\title{
A weak law of large numbers for the sequence of uncorrelated fuzzy random variables
}

\author{
Li Guan ${ }^{\mathrm{a}}$, Jinping Zhang*b, Jieming Zhou ${ }^{\mathrm{c}}$ \\ ${ }^{a}$ College of Statistics and Data Science, Faculty of Science, Beijing University of Technology,100 \\ Pingleyuan, Chaoyang District, Beijing, 100124, P.R.China \\ ${ }^{b}$ Department of Mathematics and Physics, North China Electric Power University, Beijing, 102206, \\ P.R.China \\ ${ }^{c}$ MOE-LCSM, School of Mathematics and Statistics, Hunan Normal University, Changsha, Hunan, \\ 410081, P. R. China
}

\begin{abstract}
We shall prove a weak law of large numbers for the uncorrelated (see Definition 3.1) fuzzy random variable sequence with respect to the uniform Hausdorff metric $d_{H}^{\infty}$, which is an extension of weak law of large numbers for independent fuzzy random variables.
\end{abstract}

Keywords: Fuzzy random variable, Uncorrelated, Law of large numbers

2000 MSC: Primary 60D05, Secondary 03E72, 54C65

\section{Introduction}

Limit theory is an important topic since sometimes we need to consider the asymptotic behavior or convergence property in applied fields such as stochastic control, mathematical finance, statistics, operational research and optimization etc. The law of large numbers (LLN) is the important limit theorem with wide application in solving practical problems.

Fuzzy random variable is the natural extension of random set (or set-valued random variable). A usual way to study fuzzy random variable is to consider its $\alpha$-level sets, which is a set-valued random variable for each $\alpha$. The law of large numbers for set-valued and fuzzy random variables has received much attention since Artstein and Vitale [1] proved the first LLN for compact random sets. For example, Hiai [7] in 1985 proved the SLLN (strong law of large numbers) for set-valued random variables in the Mosco convergence. Uemura [18] obtained a law of large numbers for random sets taking values in a class of subsets larger than the class of compact subsets of a Banach space. Detail review concerning LLN for set-valued random variables earlier than 2002 can be referred to the book [14]. Guan et al. [5] obtained SLLN for weighted sums of set-valued random variables in Rademacher type p Banach space. There are other references studied LLN for set-valued random variables. By using $\alpha$-level sets, some results for set-valued random variables were extended to the fuzzy case. For instance, Klement et al. [11] (1986) obtained a SLLN

Email addresses: guanli@bjut.edu.cn (Li Guan), zhangjinping@ncepu.edu.cn (Jinping Zhang*), jmzhou@hunnu.edu.cn (Jieming Zhou)

*Corresponding author: Jinping Zhang 
for independent and identically distributed compact fuzzy random variables. Inoue [9] (1991) studied SLLN for independent tight fuzzy random variables. Kim [10] proved a SLLN for independent and identically distributed fuzzy random variables using a different metric. Guan and Li [4] studied LLN for weighted sums of fuzzy random variables. Li and Ogura [13] obtained the SLLN for independent (not necessarily identical distributed) fuzzy random variables. Terán [17] studied SLLN for t-normed arithmetics.

In this paper, at first we propose the definition of uncorrelated fuzzy random variables (see Definition 3.1) in the sense of level-wise by considering the $\alpha$-level set. For two fuzzy random variables, uncorrelation is weaker than independence. Under the weaker condition, then we shall prove a weak law of large numbers for the sequence of fuzzy random variables in the real line $\mathbb{R}$ with respect to the uniform Hausdorff metric $d_{H}^{\infty}$, which is different from the existing literature.

The rest of the paper is organized as follows: Section 2 contributes to preliminaries on set-valued and fuzzy random variables. In Section 3, we shall present the main result.

\section{Preliminaries}

Throughout this paper, $(\Omega, \mathscr{A}, P)$ denotes a nonatomic complete probability space. $\mathbb{R}$ is the set of real numbers. $K(\mathbb{R})$ denotes the family of all nonempty closed subsets of $\mathbb{R}$. $K_{k}(\mathbb{R})$ is the family of all nonempty compact subsets of $\mathbb{R}$, and $K_{k c}(\mathbb{R})$ is the family of all nonempty compact convex subsets of $\mathbb{R}$.

For any $A, B \in K(\mathbb{R})$ and $\lambda \in \mathbb{R}$, the addition and scalar multiplication are defined as follows:

$$
\begin{gathered}
A+B=\{a+b: a \in A, b \in B\}, \\
\lambda A=\{\lambda a: a \in A\} .
\end{gathered}
$$

The Hausdorff metric on $K(\mathbb{R})$ is defined by

$$
d_{H}(A, B)=\max \left\{\sup _{a \in A} \inf _{b \in B}|a-b|, \sup _{b \in B} \inf _{a \in A}|a-b|\right\}
$$

for $A, B \in K(\mathbb{R})$. For $A \in K(\mathbb{R})$, define $\|A\|_{\mathbf{K}}:=d_{H}(\{0\}, A)$. It is known that the metric space $\left(K_{k}(\mathbb{R}), d_{H}\right)$ is complete and separable, and $K_{k c}(\mathbb{R})$ is a closed subset of $\left(K_{k}(\mathbb{R}), d_{H}\right)$ (cf. [14], Theorems 1.1.2 and 1.1.3).

Now we give a property of Hausdorff metric needed later, which appeared in [13] without given proof.

Proposition 2.1. Let $A_{1} \subset A_{2} \subset A_{3}$ and $B_{1} \subset B_{2} \subset B_{3}$. All of them belong to $K_{k}(\mathbb{R})$. Then we have

$$
d_{H}\left(A_{2}, B_{2}\right) \leq d_{H}\left(A_{1}, B_{3}\right)+d_{H}\left(A_{3}, B_{1}\right),
$$

where $d_{H}(x, A)=\inf _{a \in A}|x-a|$ for $A \subset \mathbb{R}$.

Proof. $d_{H}\left(A_{2}, B_{2}\right)<\infty$ since both $A_{2}$ and $B_{2}$ are compact. By virtue of Theorem 1.1.14 in [14],

$$
d_{H}\left(A_{2}, B_{2}\right)=\sup _{x \in \mathbb{R}}\left\{\left|d\left(x, A_{2}\right)-d\left(x, B_{2}\right)\right|\right\} .
$$


For $x \in \mathbb{R}$, we have

$$
d\left(x, A_{2}\right)-d_{H}\left(x, B_{2}\right) \leq d\left(x, A_{1}\right)-d\left(x, B_{3}\right)
$$

and

$$
d\left(x, A_{2}\right)-d_{H}\left(x, B_{2}\right) \geq d\left(x, A_{3}\right)-d\left(x, B_{1}\right) .
$$

Then

$$
\left|d\left(x, A_{2}\right)-d\left(x, B_{2}\right)\right| \leq \max \left\{\left|d\left(x, A_{1}\right)-d\left(x, B_{3}\right)\right|,\left|d\left(x, A_{3}\right)-d\left(x, B_{1}\right)\right|\right\} .
$$

Furthermore,

$$
\begin{aligned}
d_{H}\left(A_{2}, B_{2}\right) & =\sup _{x \in \mathbb{R}}\left\{\left|d\left(x, A_{2}\right)-d\left(x, B_{2}\right)\right|\right\} \\
& \leq \sup _{x \in \mathbb{R}} \max \left\{\left|d\left(x, A_{1}\right)-d\left(x, B_{3}\right)\right|,\left|d\left(x, A_{3}\right)-d\left(x, B_{1}\right)\right|\right\} \\
& \leq \sup _{x \in \mathbb{R}}\left\{\left|d\left(x, A_{1}\right)-d\left(x, B_{3}\right)\right|\right\}+\sup _{x \in \mathbb{R}}\left\{\left|d\left(x, A_{3}\right)-d\left(x, B_{1}\right)\right|\right\} \\
& =d_{H}\left(A_{1}, B_{3}\right)+d_{H}\left(A_{3}, B_{1}\right) .
\end{aligned}
$$

Remark 1. In general Banach space, the result also holds and further, from (11) we can get the stronger result

$$
d_{H}\left(A_{2}, B_{2}\right) \leq \max \left\{d_{H}\left(A_{1}, B_{3}\right), d_{H}\left(A_{3}, B_{1}\right)\right\},
$$

which was stated in [15] without proof.

A set-valued mapping $F: \Omega \rightarrow K(\mathbb{R})$ is called a set-valued random variable (or a random set), if for each open subset $O$ of $X$, the inverse image $F^{-1}(O):=\{\omega \in \Omega$ : $F(\omega) \cap O \neq \emptyset\}$ belongs to $\mathscr{A}$.

The family of all integrable selections of $F$ is denoted by

$$
S_{F}:=\left\{f \in L^{1}[\Omega ; \mathbb{R}]: f(\omega) \in F(\omega) \text { a.s. }\right\},
$$

where $L^{1}[\Omega ; \mathbb{R}]$ is the family of all Lebesgue integrable (with respect to $P$ ) $\mathbb{R}$-valued functions.

A set-valued random variable $F$ is called integrable if $S_{F}$ is non-empty. It is called integrably bounded if $\int_{\Omega}\|F(\omega)\|_{\mathbf{K}} d P<\infty$, which is equivalent to that $S_{F}$ is a bounded subset of $L^{1}[\Omega ; \mathbb{R}]$ (cf. [8] or [14]). $L^{1}[\Omega, \mathscr{A}, P ; K(\mathbb{R})]$ denotes the space of all integrably bounded $K(\mathbb{R})$-valued random variables. Similarly, we have notations $L^{1}\left[\Omega, \mathscr{A}, P ; K_{k}(\mathbb{R})\right]$ and $L^{1}\left[\Omega, \mathscr{A}, P ; K_{k c}(\mathbb{R})\right]$ respectively.

For $F, G \in L^{1}[\Omega, \mathscr{A}, P ; K(\mathbb{R})], F=G$ means in the sense of $F(\omega)=G(\omega)$ a.s.

For an integrable set-valued random variable $F$, its expectation, denoted by $E[F]$, is defined by Aumann in [2] as following

$$
E[F]:=\left\{\int_{\Omega} f d P: f \in S_{F}\right\},
$$


where $\int_{\Omega} f d P$ is the usual Lebesgue integral. $E[F]$ is also called the Aumann integral in literatures. Since here the underlying space is $\mathbb{R}$ and $(\Omega, \mathscr{A}, P)$ has no atom, it is known that the expectation $E[F]$ is a closed and convex subset of $\mathbb{R}$.

Let $\mathcal{F}_{k}(\mathbb{R})$ be the family of all compact fuzzy sets: $v: \mathbb{R} \rightarrow[0,1]$, where $v$ satisfies the following conditions:

(1) The 1-level set $v_{1}=\{x \in \mathbb{R}: v(x)=1\} \neq \emptyset$.

(2) $v$ is upper semicontinuous, i.e. for each $\alpha \in[0,1]$, the $\alpha$-level set $v_{\alpha}:=\{x \in \mathbb{R}$ : $v(x) \geq \alpha\}$ is a closed subset of $\mathbb{R}$.

(3) The support set $\operatorname{cl}\{x \in \mathbb{R}: v(x)>0\}$ is compact.

A fuzzy set $v$ in $\mathcal{F}_{k}(\mathbb{R})$ is called convex if it satisfies

$$
v(\lambda x+(1-\lambda) y) \geq \min \{v(x), v(y)\}, \text { for any } x, y \in \mathbb{R}, \lambda \in[0,1] .
$$

It is known that $v$ is convex if and only if each $\alpha$-level set $v_{\alpha}(\alpha \in(0,1])$ is a convex subset of $\mathbb{R}$. $\mathcal{F}_{k c}(\mathbb{R})$ denotes the class of all compact convex fuzzy sets.

The uniform metric $d_{H}^{\infty}\left(\mathrm{cf}\right.$. [16]) in $\mathcal{F}_{k}(\mathbb{R})$ is defined as follows: for $v^{1}, v^{2} \in \mathcal{F}_{k}(\mathbb{R})$,

$$
d_{H}^{\infty}\left(v^{1}, v^{2}\right):=\sup _{\alpha \in(0,1]} d_{H}\left(v_{\alpha}^{1}, v_{\alpha}^{2}\right) .
$$

Define the norm $\|v\|_{\mathbf{F}}:=d_{H}^{\infty}\left(v, I_{0}\right)=\sup _{\alpha>0}\left\|v_{\alpha}\right\|_{\mathbf{K}}$, where $I_{0}$ is the indicator function of $\{0\}$. The space $\left(\mathcal{F}_{k}(\mathbb{R}), d_{H}^{\infty}\right)$ is a complete metric space (cf. [12]) but not separable in general (cf. [14], Remark 5.1.7). Completeness was first proved by Puri and Ralescu [16] in the case of the d-dimensional Euclidean space $\mathbb{R}^{d}$.

It is well known that $v_{\alpha}=\bigcap_{\beta<\alpha} v_{\beta}$, for every $\alpha \in(0,1]$. We denote $v_{\alpha+}=c l\left(\bigcup_{\beta>\alpha} v_{\beta}\right)$, for $\alpha \in[0,1)$, which will be used later. Obviously, $v_{0+}$ is the support set of $v$. Due to the completeness of $\left(\mathcal{F}_{k}(\mathbb{R}), d_{H}^{\infty}\right)$, every Cauchy sequence $\left\{v^{n}: n \in \mathbb{N}\right\}$ converges in $\mathcal{F}_{k}(\mathbb{R})$ with respect to the metric $d_{H}^{\infty}$.

Now we present a result which will be used later.

Lemma 2.1. (cf. Lemma 2 of [13]) Suppose a sequence $\left\{v^{n}: n \in \mathbb{N}\right\}$ in $\mathcal{F}_{k}(\mathbb{R})$ converges to $v$ in $\mathcal{F}_{k}(\mathbb{R})$ with respect to $d_{H}^{\infty}$. Then for each $\alpha \in[0,1)$, the sequence $\left\{v_{\alpha+}^{n}: n \in \mathbb{N}\right\}$ converges to a set $v_{\alpha^{*}}$ in $K_{k}(\mathbb{R})$ with respect to $d_{H}$. Further, $\lim _{\beta \downarrow \alpha} d_{H}\left(v_{\beta}, v_{\alpha^{*}}\right)=0$, so that $v_{\alpha^{*}}=v_{\alpha+}$.

For any $v \in \mathcal{F}_{k c}(\mathbb{R})$, define the support function of $v$ as follows

$$
s_{v}\left(x^{*}, \alpha\right)=\left\{\begin{array}{cc}
s\left(x^{*}, v_{\alpha}\right) & \text { if } \alpha>0, \\
s\left(x^{*}, v_{0+}\right) & \text { if } \alpha=0,
\end{array}\right.
$$

for $\left(x^{*}, \alpha\right) \in S^{*} \times[0,1]$, where $S^{*}$ is the unit sphere of $\mathbb{R}^{*}\left(\mathbb{R}^{*}=\mathbb{R}\right.$ in the sense of isomorphism, but for the sake of clarity, we still use $\mathbb{R}^{*}$ later $)$ and $s\left(x^{*}, A\right)=\sup _{a \in A} x^{*}(a)$ for $x^{*} \in S^{*}$ and $A \subset \mathbb{R}$.

A mapping $X: \Omega \rightarrow \mathcal{F}(\mathbb{R})$ is called a fuzzy set-valued random variable or a random upper semicontinuous function, if, for every $\alpha \in(0,1], X_{\alpha}(\omega)=\{x \in \mathbb{R}: X(\omega)(x) \geq \alpha\}$ is a set-valued random variable.

A fuzzy random variable $X$ is called integrably bounded if the real-valued random variable $\left\|X_{0+}(\omega)\right\|_{\mathbf{K}}$ is integrable. Let $L^{1}\left[\Omega, \mathscr{A}, P ; \mathcal{F}_{k}(\mathbb{R})\right]$ be the set of all integrably bounded 
fuzzy random variables and $L^{1}\left[\Omega, \mathscr{A}, P ; \mathcal{F}_{k c}(\mathbb{R})\right]$ be the set of all integrably bounded fuzzy random variables taking values in $\mathcal{F}_{k c}(\mathbb{R})$. Two fuzzy random variables $X, Y \in$ $L^{1}\left[\Omega, \mathscr{A}, P ; \mathcal{F}_{k}(\mathbb{R})\right]$ are considered to be identical if for any $\alpha \in[0,1], X_{\alpha}(\omega)=Y_{\alpha}(\omega)$ a.s.

The expectation of a fuzzy random variable $X$, denoted by $E[X]$, is an element in $\mathcal{F}_{k}(\mathbb{R})$ such that, for every $\alpha \in(0,1]$,

$$
(E[X])_{\alpha}=E\left[X_{\alpha}\right]
$$

where the expectation of right hand side is the Aumann integral. From the existence theorem (cf. [12]), we can get an equivalent definition: for any $x \in \mathbb{R}$,

$$
E(X)(x)=\sup \left\{\alpha \in[0,1]: x \in E\left[X_{\alpha}\right]\right\} .
$$

Note that $E[X]$ is always convex since $(\Omega, \mathscr{A}, P)$ is nonatomic.

\section{Main Results}

Definition 3.1. Let $X^{1}, X^{2}$ be fuzzy random variables. $X^{1}$ and $X^{2}$ are called uncorrelated if for any $\alpha \in(0,1], X_{\alpha}^{1}$ and $X_{\alpha}^{2}$ are uncorrelated set-valued random variables. I.e. for each $x^{*} \in \mathbb{R}^{*}$, the real-valued random variables $s\left(x^{*}, X_{\alpha}^{1}\right)$ and $s\left(x^{*}, X_{\alpha}^{2}\right)$ are uncorrelated in the usual sense.

Fuzzy random variables sequence $X^{1}, X^{2}, \cdots$ are called uncorrelated if the sequence $X_{\alpha}^{1}, X_{\alpha}^{2}, \cdots$ are pairwise uncorrelated for any $\alpha \in(0,1]$.

Lemma 3.1. Let $\left\{X^{n}: n \in \mathbb{N}\right\}$ be a sequence of uncorrelated $\mathcal{F}_{k c}(\mathbb{R})$-valued random variables. Then for each $\alpha \in(0,1],\left\{X_{\alpha+}^{n}: n \in \mathbb{N}\right\}$ is a sequence of uncorrelated $K_{k c}(\mathbb{R})$ valued random variables.

Proof. Take a decreasing sequence $\left\{\alpha_{j}\right\} \subset(0,1]$ such that it converges to $\alpha$. Then $X_{\alpha+}^{n}=$ $\operatorname{cl}\left(\bigcup_{j} X_{\alpha_{j}}^{n}\right), X_{\alpha+}^{m}=\operatorname{cl}\left(\bigcup_{j} X_{\alpha_{j}}^{m}\right)$ for $m, n \in \mathbb{N}$. By Lemma 2.1, it holds that

$$
\lim _{j} d_{H}\left(X_{\alpha_{j}}^{n}, X_{\alpha+}^{n}\right)=0, \quad \lim _{j} d_{H}\left(X_{\alpha_{j}}^{m}, X_{\alpha+}^{m}\right)=0 .
$$

Furthermore, for each $x^{*} \in \mathbb{R}^{*}$

$$
\lim _{j} s\left(x^{*}, X_{\alpha_{j}}^{n}\right)=s\left(x^{*}, X_{\alpha+}^{n}\right), \quad \lim _{j} s\left(x^{*}, X_{\alpha_{j}}^{m}\right)=s\left(x^{*}, X_{\alpha+}^{m}\right) .
$$

By Definition 3.1, we know that

$$
\operatorname{Cov}\left(s\left(x^{*}, X_{\alpha}^{n}\right), s\left(x^{*}, X_{\alpha}^{m}\right)\right)=0 .
$$

Therefore, by the monotone convergence theorem, we have

$$
\begin{aligned}
\operatorname{Cov}\left(s\left(x^{*}, X_{\alpha+}^{n}\right), s\left(x^{*}, X_{\alpha+}^{m}\right)\right) & =\operatorname{Cov}\left(\lim _{j} s\left(x^{*}, X_{\alpha_{j}}^{n}\right), \lim _{j} s\left(x^{*}, X_{\alpha_{j}}^{m}\right)\right) \\
& =\lim _{j} \operatorname{Cov}\left(s\left(x^{*}, X_{\alpha_{j}}^{n}\right), s\left(x^{*}, X_{\alpha_{j}}^{m}\right)\right) \\
& =0 .
\end{aligned}
$$

That shows the uncorrelation of the sequence $\left\{X_{\alpha+}^{n}: n \in \mathbb{N}\right\}$ for any $\alpha \in(0,1]$. 
Theorem 3.1. Let $\left\{X^{n}: n \in \mathbb{N}\right\}$ be a sequence of uncorrelated $\mathcal{F}_{k c}(\mathbb{R})$-valued random variables such that for each $n, \operatorname{Var}\left(s\left(x^{*}, X_{\alpha}^{n}\right)\right)$ exists and for any $x^{*} \in \mathbb{R}^{*}$,

$$
\frac{1}{n^{2}} \sum_{k=1}^{n} \operatorname{Var}\left(s\left(x^{*}, X_{\alpha}^{k}\right)\right) \longrightarrow 0 \text { as } n \rightarrow \infty \text {. }
$$

Then

$$
P\left\{d_{H}^{\infty}\left(\frac{1}{n} \sum_{k=1}^{n} X^{k}, \frac{1}{n} \sum_{k=1}^{n} E\left[X^{k}\right]\right)>\varepsilon\right\} \longrightarrow 0 \text { as } n \rightarrow \infty .
$$

Proof. step 1:

Firstly we prove that for any $\alpha \in(0,1]$,

$$
\frac{1}{n^{2}} \sum_{k=1}^{n} \operatorname{Var}\left(s\left(x^{*}, X_{\alpha+}^{k}\right)\right) \longrightarrow 0 \text { as } n \rightarrow \infty \text {. }
$$

Take a decreasing sequence $\left\{\alpha_{j}\right\} \subset(0,1]$, which converges to $\alpha . X_{\alpha+}^{n}=\operatorname{cl}\left(\bigcup_{j} X_{\alpha_{j}}^{n}\right)$. By Lemma 2.1, we have

$$
\lim _{j} d_{H}\left(X_{\alpha_{j}}^{n}, X_{\alpha+}^{n}\right)=0
$$

and

$$
\lim _{j} s\left(x^{*}, X_{\alpha_{j}}^{n}\right)=s\left(x^{*}, X_{\alpha+}^{n}\right) .
$$

Therefore, by the monotone convergence theorem and the condition (2), we have

$$
\begin{aligned}
\frac{1}{n^{2}} \sum_{k=1}^{n} \lim _{j} \operatorname{Var}\left(s\left(x^{*}, X_{\alpha_{j}}^{n}\right)\right) & =\frac{1}{n^{2}} \sum_{k=1}^{n} \operatorname{Var}\left(s\left(x^{*}, X_{\alpha+}^{n}\right)\right) \\
& \longrightarrow 0 \text { as } n \rightarrow \infty .
\end{aligned}
$$

By Lemma 3.1, we know that both $\left\{X_{\alpha}^{n}: n \in \mathbb{N}\right\}$ and $\left\{X_{\alpha+}^{n}: n \in \mathbb{N}\right\}$ are sequences of uncorrelated set-valued random variables. Then by theorem 3.2 of [] $]$, we have

$$
P\left\{d_{H}\left(\frac{1}{n} \sum_{k=1}^{n} X_{\alpha}^{k}, \frac{1}{n} \sum_{k=1}^{n} E\left[X_{\alpha}^{k}\right]\right)>\varepsilon\right\} \longrightarrow 0 \text { as } n \rightarrow \infty
$$

and

$$
P\left\{d_{H}\left(\frac{1}{n} \sum_{k=1}^{n} X_{\alpha+}^{k}, \frac{1}{n} \sum_{k=1}^{n} E\left[X_{\alpha+}^{k}\right]\right)>\varepsilon\right\} \longrightarrow 0 \text { as } n \rightarrow \infty
$$

step 2:

Take $\varepsilon>0$, and apply Lemma 2 for $v_{n}=\frac{1}{n} \sum_{i=1}^{n} E\left[X^{i}\right]$. Then we can find a sequence $0=\alpha_{0}<\alpha_{1}<\ldots<\alpha_{m}=1$ such that

$$
d_{H}\left(\left(\frac{1}{n} \sum_{i=1}^{n} E\left[X^{i}\right]\right)_{\alpha_{k}},\left(\frac{1}{n} \sum_{i=1}^{n} E\left[X^{i}\right]\right)_{\alpha_{k-1}+}\right)<\varepsilon .
$$


Hence, by virtue of monotone property of level sets and the above results, we have

$$
\left(\frac{1}{n} \sum_{i=1}^{n} X^{i}\right)_{\alpha}=\frac{1}{n} \sum_{i=1}^{n} X_{\alpha}^{i},\left(\frac{1}{n} \sum_{i=1}^{n} E\left[X^{i}\right]\right)_{\alpha}=\frac{1}{n} \sum_{i=1}^{n} E\left[X_{\alpha}^{i}\right],
$$

For $\alpha_{k-1}<\alpha \leq \alpha_{k}$, we have

$$
\left(\frac{1}{n} \sum_{i=1}^{n} X^{i}\right)_{\alpha_{k-1}+} \supseteq\left(\frac{1}{n} \sum_{i=1}^{n} X^{i}\right)_{\alpha} \supseteq\left(\frac{1}{n} \sum_{i=1}^{n} X^{i}\right)_{\alpha_{k}}
$$

and

$$
\left(\frac{1}{n} \sum_{i=1}^{n} E\left[X^{i}\right]\right)_{\alpha_{k-1}+} \supseteq\left(\frac{1}{n} \sum_{i=1}^{n} E\left[X^{i}\right)\right]_{\alpha} \supseteq\left(\frac{1}{n} \sum_{i=1}^{n} E\left[X^{i}\right]\right)_{\alpha_{k}} .
$$

Then by Proposition 2.1, we obtain

$$
\begin{aligned}
& d_{H}\left(\left(\frac{1}{n} \sum_{i=1}^{n} X^{i}\right)_{\alpha},\left(\frac{1}{n} \sum_{i=1}^{n} E\left[X^{i}\right]\right)_{\alpha}\right) \\
& \leq d_{H}\left(\left(\frac{1}{n} \sum_{i=1}^{n} X^{i}\right)_{\alpha_{k}},\left(\frac{1}{n} \sum_{i=1}^{n} E\left[X^{i}\right]\right)_{\alpha_{k-1}+}\right) \\
& \quad+d_{H}\left(\left(\frac{1}{n} \sum_{i=1}^{n} X^{i}\right)_{\alpha_{k-1}+},\left(\frac{1}{n} \sum_{i=1}^{n} E\left[X^{i}\right]\right)_{\alpha_{k}}\right) \\
& \leq d_{H}\left(\frac{1}{n} \sum_{i=1}^{n} X_{\alpha_{k}}^{i}, \frac{1}{n} \sum_{i=1}^{n} E\left[X^{i}\right]_{\alpha_{k}}\right) \\
& +d_{H}\left(\frac{1}{n} \sum_{i=1}^{n} X_{\alpha_{k-1}+}^{i}, \frac{1}{n} \sum_{i=1}^{n} E\left[X^{i}\right]_{\alpha_{k-1}+}\right) \\
& \quad+2 d_{H}\left(\left(\frac{1}{n} \sum_{i=1}^{n} E\left[X^{i}\right]\right)_{\alpha_{k}},\left(\frac{1}{n} \sum_{i=1}^{n} E\left[X^{i}\right]\right)_{\alpha_{k-1}+}\right) .
\end{aligned}
$$

Consequently,

$$
\begin{aligned}
& d_{H}^{\infty}\left(\frac{1}{n} \sum_{i=1}^{n} X^{i}, \frac{1}{n} \sum_{i=1}^{n} E\left[X^{i}\right]\right) \\
& =\sup _{\alpha \in(0,1]} d_{H}\left(\left(\frac{1}{n} \sum_{i=1}^{n} X^{i}\right)_{\alpha},\left(\frac{1}{n} \sum_{i=1}^{n} E\left[X^{i}\right]\right)_{\alpha}\right) \\
& \leq \max _{1 \leq k \leq m} d_{H}\left(\frac{1}{n} \sum_{i=1}^{n} X_{\alpha_{k}}^{i}, \frac{1}{n} \sum_{i=1}^{n} E\left[X_{\alpha_{k}}^{i}\right]\right) \\
& \quad+\max _{1 \leq k \leq m} d_{H}\left(\frac{1}{n} \sum_{i=1}^{n} X_{\alpha_{k-1}+}^{i}, \frac{1}{n} \sum_{i=1}^{n} E\left[X_{\alpha_{k-1}+}^{i}\right]\right) \\
& +2 \max _{1 \leq k \leq m} d_{H}\left(\left(\frac{1}{n} \sum_{i=1}^{n} E\left[X^{i}\right]\right)_{\alpha_{k}},\left(\frac{1}{n} \sum_{i=1}^{n} E\left[X^{i}\right]\right)_{\alpha_{k-1}+}\right)
\end{aligned}
$$


By using (44), (5) and (6), for any given positive number $\varepsilon$, we obtain

$$
P\left\{d_{H}^{\infty}\left(\frac{1}{n} \sum_{k=1}^{n} X^{k}, \frac{1}{n} \sum_{k=1}^{n} E\left[X^{k}\right]\right)>\varepsilon\right\} \longrightarrow 0 \text { as } n \rightarrow \infty .
$$

\section{Concluding remark}

As a manner similar to the uncorrelated set-valued random variables, we proposed uncorrelated fuzzy random variables by considering the $\alpha$-level sets. Uncorrelation is weaker than independence. For the sequence of uncorrelated fuzzy random variables, we proved a weak law of large numbers, which is an extension of weak law of large numbers for independent fuzzy random variables. With the development of technology, complex and big data are produced and obtained. Fuzzy statistics is a nice tool to deal with complex data. We wish our result will be applicable in fuzzy statistics.

\section{Acknowledgment}

This work is partly supported by the National Social Science Fund of China No.19BTJ017(Li Guan), Natural Science Foundation of Beijing Municipality (No.1192015) (Jinping Zhang).

\section{References}

[1] Artstein, Z. and Vitale, R.A.: A strong law of large numbers for random compact sets. Ann. Probab. 3, 879-882. (1975)

[2] Aumann, R. : Integrals of set-valued functions. J. Math. Anal. Appl., 12, 1-12. (1965)

[3] Castaing, C. and Valadier, M.: Convex Analysis and Measurable Multifunctions, Lect. Notes in Math. 580, Springer-Verlag, Berlin, New York. (1977)

[4] Guan, L. and Li, S.: Laws of large numbers for weighted sums of fuzzy set-valued random variables, Inter. J. of Uncertainty, Fuzziness and Knowledge-based System Vol.12, 811-825. (2004)

[5] Guan, L. Li, S. and Inoue, H.: Strong laws of large numbers for weighted sums of setvalued random variables in Rademacher type p Banach space, Scientiae Mathematicae Japonicae, 67 (3), 377-392. (2007)

[6] Guan, L. and Zhang, J.: Laws of Large Numbers for Uncorrelated Set-Valued Random Variables, arXiv:2011.07199 [math.PR] (2020)

[7] Hiai, F.: Convergence of conditional expectations and strong laws of large numbers for multivalued random variables, Trans. A.M.S., 291, 613-627. (1985)

[8] Hiai, F. and Umegaki, H.: Integrals, conditional expectations and martingales of multivalued functions. Jour. Multivar. Anal., 7, 149-182. (1977) 
[9] Inoue, H.: A strong law of large numbers for fuzzy random sets. Fuzzy Sets and Systems, 41, 285-291. (1991)

[10] Kim, Y.K.: A strong law of large numbers for fuzzy random variables, Fuzzy Sets and Systems, 111, 319-323. (2000)

[11] Klement, E. P., Puri, M. L. and Ralescu, D. A.: Limit theorems for fuzzy random variables. Proc. Roy. Soc. Lond. A., 407, 171-182. (1986)

[12] Li, S. and Ogura, Y.: Fuzzy random variables, conditional expectations and fuzzy martingales. J. Fuzzy Math. 4, 905-927. (1996)

[13] Li, S. and Ogura, Y.: Strong laws of numbers for independent fuzzy set-valued random variables, Fuzzy Sets and Systems, 157, 2569-2578. (2006)

[14] Li, S., Ogura, Y. and Kreinovich, V.: Limit Theorems and Applications of Set-Valued and Fuzzy Sets-Valued Random Variables. Kluwer Academic Publishers (2002)

[15] Molchanov, I. S. On strong laws of large numbers for random upper semicontinuous functions, Fuzzy Sets and Systems, 235, 349-355. (1999)

[16] Puri, M. L. and Ralescu, D. A.: Fuzzy random variables. J. Math. Anal. Appl., 114, 406-422. (1986)

[17] Terán, P.: Strong law of large numbers for t-normed arithmetics. Fuzzy Sets and Systems, 159, 343-360. (2008)

[18] Uemura, T.: A law of large numbers for random sets. Fuzzy Sets and Systems, 59, 181-188. (1993) 